\title{
Jump Adapted Scheme Of a Non Mark Dependent Jump Diffusion Process with Application to the Merton Jump Diffusion Model
}

\author{
Renaud Fadonougbo ${ }^{1} \&$ George O. Orwa ${ }^{2}$ \\ ${ }^{1}$ Pan African University Institute for Basic Sciences Technology and Innovation, Kenya \\ ${ }^{2}$ Statistics and Actuarial Department, Jomo Kenyatta University of Agriculture and Technology, Nairobi, Kenya \\ Correspondence: Renaud Fadonougbo, Pan African University Institute for Basic Sciences Technology and Innovation, \\ Kenya. E-mail: renaudfadonougbo@gmail.com
}

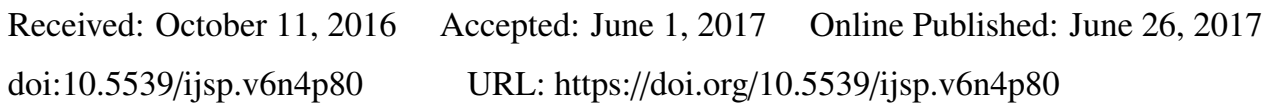

The research is financed by (Pan African University)

\begin{abstract}
This paper provides a complete proof of the strong convergence of the Jump adapted discretization Scheme in the univariate and mark independent jump diffusion process case. We put in detail and clearly a known and general result for mark dependent jump diffusion process. A Monte-Carlo simulation is used as well to show numerical evidence.
\end{abstract}

Keywords: jump-diffusion process,jump-adapted discretization, strong convergence, non-dependent mark, Monte-Carlo simulation

\section{Introduction}

Since three decades, extensive research have been conducted on the dynamics of asset prices, bond etc, and there is no more doubt about the evidence of presence of jumps, see (Johannes, 2004; Akgiray \& Booth, 1988; Glasserman \& kou, 2003). The jump diffusion modeling was first introduced by (Merton, 1976) for option pricing. He showed that asset dynamics present discontinuous path instead of continuous one as claimed in Black-Scholes derivation, see (Black $\&$ Scholes, 1973), (Cox, Ingersoll, \& Ross, 1985) and (Vasicek, 1977). The jump process, now became popular as it drew more attention and being very useful as modeling frame in many area of finance such as credit risk modeling, interest rate, and in the computation of Value at Risk (Var), see (Johannes, 2004; Akgiray \& Booth, 1988; Glasserman \& Kou, 2003) and (Aï-Sahalia \& Yu, 2006). It is also used to describe some stylized facts presented by financial data, see (Glasserman, 2004, page 134). Regardless of the importance of the jump diffusion process, its implementation remains a challenge. The process is always described as solution of a given stochastic differential equation(SDE), but it is only known in its explicit form just in few cases, and their probability distribution is not always known see (Aït-Sahalia, Fan, \& Peng, 2009) and (Yu, 2007). That complexity has led researchers to conduct extensive research of finding appropriate way to approximate the stochastic process. Such approximation is essentially based on discretization approach. As discretization approach there are two range methods of discretization of jump diffusion processes which are the weak convergence approximation and the strong convergence approximation, see details in (Bruti-Liberati \& Platen, 2007). In this work we are interested in one type of strong convergence approximation, called Jump adapted discretization as it offers a simple computation and allows easily an extension of discretization methods known for diffusion processes. Based on (Bruti-Liberati \& Platen2007) convergence theorem, we derived similar result for one dimension Jump adapted approximation with a non dependent Mark jump with a compound Poisson process.

\subsection{Jump Diffusion Model}

The model under study is a univariate parametric jump diffusion process defined on the probability space $\left(\Omega, \mathcal{F}_{T}, \mathcal{F}, P\right)$ as:

$$
d X_{t}=\mu\left(X_{t}, \theta\right) d t+\sigma\left(X_{t}, \theta\right) d W_{t}+J\left(X_{t}\right) d N_{t}
$$

with, $X_{t}$ the vector of dimension $n=1$ that represents the state, $W_{t}$ a standard Brownian motion of dimension $d=1$, $\mu\left(X_{t}, \theta\right): \mathbb{R}^{n} \rightarrow \mathbb{R}^{n}$ is the drift, $\sigma\left(X_{t}, \theta\right): \mathbb{R}^{n} \rightarrow \mathbb{R}^{n \times d}$ is called diffusion and the variance matrix of the process can be defined as: $V(x, \theta)=\sigma\left(X_{t}, \theta\right) \sigma\left(X_{t}, \theta\right)^{T 1}$.

$N_{t}$ the pure jump process whose intensity is $\lambda(\theta), J_{t}$ is the jump size with probability density $v(., \theta)$, whose support is $C \subset \mathbb{R}^{n}$ and $\theta \in \Theta$, a compact subset of $\mathbb{R}^{k}$, that parameterizes the model. To be consistent we have to differentiate the values of the process before and after the occurrence of a potential jump. Therefore we will denote by $X_{t-}$ and $J_{t-}$ the value and the size respectively of the process and the jump before the occurrence of jump. Thus 1 can be rewritten as

\footnotetext{
${ }^{1} \sigma\left(X_{t}, \theta\right)^{T}$ is the transposition of $\sigma\left(X_{t}, \theta\right)$
} 
follows:

$$
d X_{t}=\mu\left(X_{t^{-}}, \theta\right) d t+\sigma\left(X_{t^{-}}, \theta\right) d W_{t}+J\left(X_{t-}\right) d N_{t}
$$

where $X_{t-}=\lim _{\vec{u}<t} X_{u}$. Let us made the following conditions on the coefficient functions.

- Lipschitz conditions

$$
\begin{gathered}
\left|\mu(x(t), \theta)-\mu\left(y_{t}, \theta\right)\right|^{2} \leq C_{1}\left|x_{t}-y_{t}\right|^{2},\left|\sigma\left(x_{t}, \theta\right)-\sigma\left(y_{t}, \theta\right)\right|^{2} \leq C_{2}\left|x_{t}-y_{t}\right|^{2}, \\
\left|J\left(x_{t}\right)-J\left(y_{t}\right)\right|^{2} \leq C_{3}\left|x_{t}-y_{t}\right|^{2},
\end{gathered}
$$

for all $t \in[0, T]$ and $x, y \in \mathbb{R}^{d}$

- Linear growth conditions

$$
\begin{gathered}
\left|\mu\left(x_{t}, \theta\right)\right|^{2} \leq K_{1}\left(1+|x|^{2}\right),\left|\sigma\left(x_{t}, \theta\right)\right|^{2} \leq K_{2}\left(1+|x|^{2}\right), \\
\left|J\left(x_{t},\right)\right|^{2} \leq K_{3}\left(1+|x|^{2}\right),
\end{gathered}
$$

for all $t \in[0, T]$ and $x, \in \mathbb{R}^{d}$.

Therefore under conditions(3) and (4) the SDE(1)admits a unique strong solution see (Bruti-Liberati-Platen, 2007). Here are some examples of univariate jump diffusion process.

1 One of the basic example of univariate jump diffusion process is as follows:

$$
d X_{t}=\mu d t+\sigma d W_{t}+J_{t} d N_{t}
$$

where, $N_{t}$ is a Poisson process with arrival rate $\lambda, J_{t}$, i.i.d. $N\left(\mu_{j}, \sigma_{j}^{2}\right)$ the jump size, $\mu\left(X_{t}, \theta\right)=\mu ; \sigma\left(X_{t}, \theta\right)=\sigma$ and $\theta=\left(\mu, \sigma, \lambda, \mu_{j}, \sigma_{j}\right)$. The conditional distribution is well known and it is as follows:

$$
X_{t} \mid X_{s}=x \rightsquigarrow N\left(x+\mu(t-s)+j \mu_{j}, \sigma^{2}(t-s)+j \sigma_{j}^{2}\right) .
$$

2 The following is the Merton jump diffusion process described as follows:

$$
d S_{t}=\mu S_{t-} d t+\sigma S_{t-} d W_{t}+S_{t-} d J_{t}
$$

with $J_{t}=\sum_{j=1}^{N_{t}}\left(Y_{j}-1\right)$, where $Y 1, Y_{2} \ldots$ are i.i.d. random variables. The solution of the $\mathrm{SDE}(6)$ is known and it is $S_{t}=S_{0} e^{\left(\mu-\frac{1}{2}\right) t+\sigma W(t)} \prod_{j=1}^{N_{t}} Y_{j}$ see (Merton, 1976).

\subsection{Discretization Review Method}

The transition density has an analytic expression for a few cases, and in general no, many approaches have proposed to get an approximation. In fact most of the method have been proposed for diffusion process. Since the jump diffusion process in the absence of jump remains a diffusion process is better to have an overlook of these different methods.

As first method, we have the Euler scheme approximation for which the diffusion process $X_{t}$ solution of the following (SDE):

$$
d X_{t}=\mu\left(X_{t}, \theta\right) d t+\sigma\left(X_{t}, \theta\right) d W_{t}
$$

is approximated by the following discrete time model:

$$
X_{i h}=X_{(i-1) h}-\mu\left(X_{(i-1) h}, \theta\right)+\sigma\left(X_{(i-1) h}, \theta\right)+\sqrt{h} \varepsilon_{i}
$$

where $\varepsilon_{i} \sim$ i.i.dN $(0,1)$. Meanwhile of how easy it is to implement the closed form expression of the transition, the accuracy of this approximation depends on the interval observation $h$. When it is small, the approximation is accurate, but when it is large the approximation can be poor.

(Merton, 1980; Lo, 1988) have found out that the approach is not consistent when $h$ is fixed. A closely approach has been suggested by (Gollnick, Houthakker, \& Taylor, 1968) and (Bergstrom, 1966), and it is defined on integrating the SDE and the use of the following trapezoidal rule approximation:

$$
\int_{(i-1) h}^{i h} \mu\left(X_{t}, \theta\right) d t=\frac{h}{2}\left\{\mu\left(X_{i h}, \theta\right)+\mu\left(X_{(i-1) h}, \theta\right)\right\}
$$


The two approximations are equivalent when $h$ is small. Unfortunately the consistency of the approach is measured in term of $h$ and some authors have showed the asymptotic bias in the estimate is $O\left(h^{2}\right)$.

Therefore many works have been done to reduce the discretization bias on the Euler's approximation, see (Elerian, 1998) and (Tse, Zhang, \& Yu, 2004) that have improved the method of (Milstein, 1979). The first used a second order term in a stochastic Taylor series expansion has been used to refine the approximation (8), while the later in a Bayesian context improved the Milstein's scheme.

(Merener, 2004) argued that the Euler scheme converges weakly with order one while the Milstein scheme with order two. They found that between the time of ocurence of jumps, the dynamics of process are purely diffusive and it is possile to be simulated using standard discretization methods. Under conditions on the coefficient functions of the jump-diffusion process, they showed that the method used conserves the same weak convergence order for both jump diffusion and pure diffusion processes and the construction of jumps does not degrade the convergence of the method.

The following section describes the discretization approach, futher the proof of convergence is presented in section and finally the section 3 has been consecrated to some example and numerical results using Monte-Carlo approach.

\section{Jump Adapted Scheme}

The discretization approach used is called Jump-diffusion adapted approximation(JAD) that consists of separating the diffusion part from the jump one. An appropriate discretization is used for the diffusion part where the effect of the jump is added at due time. This method is based on Itô-Taylor expansion of the drift and diffusion term regardless of the jump part, see (Bruti-Liberati \& Platen2007). Since we assumed that the jump occurs at discrete point time and between two discretization points the process has a diffusion dynamics. We consider a jump time dicretization $0=t_{0}<t_{1}<\ldots<t_{N}=T$; constructed by a superposition of the jump times $\left\{\tau_{1}, \ldots\right\}$ to a deterministic equidistant grid with maximum step size $\Delta>0$. From the $\operatorname{SDE}(2)$ and for $t \in\left[t_{i}, t_{i+1}\right)$ we have :

$$
\int_{t_{i}}^{t_{i+1}} d X_{t}=\int_{t_{i}}^{t_{i+1}} \mu\left(X_{t-}, \theta\right) d t+\int_{t_{i}}^{t_{i+1}} \sigma\left(X_{t-}, \theta\right) d W_{t}+\int_{t_{i}}^{t_{i+1}} J_{t-} d N_{t}
$$

As $X_{t-}$ denotes the value of the process just before the occurrence of a potential jump, it may be then expressed as a solution of the following SDE:

$$
d X_{s}=\mu\left(X_{s}, \theta\right) d t+\sigma\left(X_{s}, \theta\right) d W_{s},
$$

and applying the Itô formula, this leads to:

$$
\begin{aligned}
\mu\left(X_{t}, \theta\right)= & \mu\left(X_{t_{i}}, \theta\right)+\int_{t_{i}}^{t} \mu_{x}\left(X_{s}, \theta\right) \mu\left(X_{s}, \theta\right) d s+\frac{1}{2} \int_{t_{i}}^{t} \mu_{x x}\left(X_{s}, \theta\right) \sigma^{2}\left(X_{s}, \theta\right) d s \\
& +\int_{t_{i}}^{t} \mu_{x}\left(X_{s}, \theta\right) \sigma\left(X_{s}, \theta\right) d W_{s}
\end{aligned}
$$

and

$$
\begin{aligned}
\sigma\left(X_{t}, \theta\right)= & \sigma\left(X_{t_{i}}, \theta\right)+\int_{t_{i}}^{t} \sigma_{x}\left(X_{s}, \theta\right) \mu\left(X_{s}, \theta\right) d s+\frac{1}{2} \int_{t_{i}}^{t} \sigma_{x x}\left(X_{s}, \theta\right) \sigma^{2}\left(X_{s}, \theta\right) d s \\
& +\int_{t_{i}}^{t} \sigma_{x}\left(X_{s}, \theta\right) \sigma\left(X_{s}, \theta\right) d W_{s}
\end{aligned}
$$

Subtituting $\mu\left(X_{t}, \theta\right)$ and $\sigma\left(X_{t}, \theta\right)$ into (10) we have:

$$
\begin{aligned}
X_{t_{i+1}}= & X_{t_{i}} \\
& +\int_{t_{i}}^{t_{i+1}} \mu\left(X_{t_{i}}, \theta\right) d t+\int_{t_{i}}^{t_{i+1}} \sigma\left(X_{t_{i}}, \theta\right) d W_{t}+\int_{t_{i}}^{t_{i+1}} \int_{t_{i}}^{t} \sigma_{x}\left(X_{s}, \theta\right) \sigma\left(X_{s}, \theta\right) d W_{s} d W_{t} \\
& +\int_{t_{i}}^{t_{i+1}} \int_{t_{i}}^{t}\left[\mu_{x}\left(X_{s}, \theta\right) \mu\left(X_{s}, \theta\right)+\frac{1}{2}\left(\mu_{x x}\left(X_{s}, \theta\right) \sigma^{2}\left(X_{s}, \theta\right)\right)\right] d s d t \\
& +\int_{t_{i}}^{t_{i+1}} \int_{t_{i}}^{t}\left[\sigma_{x}\left(X_{s}, \theta\right) \mu\left(X_{s}, \theta\right)+\frac{1}{2}\left(\sigma_{x x}\left(X_{s}, \theta\right) \sigma^{2}\left(X_{s}, \theta\right)\right)\right] d s d W_{t} \\
& +\int_{t_{i}}^{t_{i+1}} \int_{t_{i}}^{t} \mu_{x}\left(X_{s}, \theta\right) \sigma\left(X_{s}, \theta\right) d W_{s} d t+\int_{t_{i}}^{t_{i+1}} J_{t-} d N_{t} \\
= & X_{t_{i+1}-}+\int_{t_{i}}^{t_{i+1}} J_{t-} d N_{t}
\end{aligned}
$$


From (11) we deduce the following approximation $Y^{\Delta}=\left\{Y_{t_{i}}, i=0, \ldots, N\right\}$ of $X$, where:

$$
Y_{t_{i+1}}=Y_{t_{i+1}-}+\int_{t_{i}}^{t_{i+1}} J\left(Y_{t_{i+1}-}\right) d N_{t}
$$

where

$$
\begin{aligned}
Y_{t_{i+1}-} & =Y_{t_{i}}+\int_{t_{i}}^{t_{i+1}} \mu\left(Y_{t_{i}}, \theta\right) d t+\int_{t_{i}}^{t_{i+1}} \sigma\left(Y_{t_{i}}, \theta\right) d W_{t}+\int_{t_{i}}^{t_{i+1}} \int_{t_{i}}^{t} \sigma_{x}\left(Y_{s}, \theta\right) \sigma\left(Y_{s}, \theta\right) d W_{s} d W_{t} \\
& =Y_{t_{i}}+\mu\left(Y_{t_{i}}, \theta\right) h+\sigma\left(Y_{t_{i}}, \theta\right) \sqrt{h} \varepsilon_{i}+\frac{1}{2} \sigma_{x}\left(Y_{t_{i}}, \theta\right) \sigma\left(Y_{t_{i}}, \theta\right) h\left(\varepsilon_{i}^{2}-1\right)
\end{aligned}
$$

$Y^{\Delta}$ is a jump adapted scheme and it converges strongly at time $t$ with order one to the solution $X$ of the SDE(2), the proof is given in the following section.

\section{Convergence}

Before we state the result of the convergence, we have to define a certain compact notation that we use to make explicit the proof.

For $m \in \mathbb{N}$, we define the set of all multi-indices $\alpha$ as follows:

$\mathcal{M}_{m}=\left\{\alpha=\left(j_{1}, j_{2}, \ldots, j_{l}\right) ; j_{i} \in\{0,1, \ldots, m\} ; i \in\{1, \ldots, l\}\right.$, for $\left.l \in \mathbb{N}\right\} \cup\{v\}$ where $v$ is the multi-index of length zero. $n(\alpha)$ the number of component of $\alpha$ that equals to $0, \alpha$ - denotes the multi index obtained by deleting the last component of $\alpha,-\alpha$ is the one obtained by the deleting the first component of $\alpha$.

Let $\overline{\mathcal{L}}^{0}$ be the set of functions $f(t, x):[0, T] \times \mathbb{R}^{d} \longrightarrow \mathbb{R}^{d}$ from $C^{1,2}$ and $\overline{\mathcal{L}}^{k}$ the set of $f(t, x)$ with partial derivatives $\frac{\partial}{\partial x^{i}} f(t, x), i \in\{1, \ldots, d\}$, we also introduce the following operator for a function $f(t, x) \in \overline{\mathcal{L}}^{k}$

$$
\bar{L}^{(0)} f(t, x):=\frac{\partial}{\partial t} f(t, x)+\sum_{i=1}^{d} \mu^{i}(t, x) \frac{\partial}{\partial x^{i}} f(t, x)+\frac{1}{2} \sum_{i, r=1}^{d} \sum_{j=1}^{m} \sigma^{i, j} \sigma^{r, j} \frac{\partial^{2}}{\partial x^{i} \partial x^{j}} f(t, x)
$$

and

$$
\bar{L}^{(k)} f(t, x):=\sum_{i=1}^{d} \sigma^{i, k} \frac{\partial}{\partial x^{i}} f(t, x), \mathrm{k} \quad \in\{1, \ldots, m\} \quad \forall t \in[0, T] \operatorname{and} x \in \mathbb{R}^{d}
$$

The Itô coefficient function $\bar{f}_{\alpha}$ for all $\alpha \in \mathcal{M}_{m}$ and for $f(t, x):[0, T] \times \mathbb{R}^{d} \rightarrow \mathbb{R}^{d}$ is defined by:

$$
\bar{f}_{\alpha}(t, x):=\left\{\begin{array}{llr}
f(t, x) & \text { for } & l(\alpha)=0 \\
f(t, \mu(t, x)) & \text { for } & l(\alpha)=1, j_{1}=0 \\
f\left(t, \sigma^{j_{1}}(t, x)\right) & \text { for } & l(\alpha)=1, j_{1} \in\{1, \ldots, m\} \\
\bar{L}^{\left(j_{1}\right)} \bar{f}_{-\alpha}(t, x) & \text { for } & l(\alpha) \geq 2, j_{1} \in\{0, \ldots, m\}
\end{array}\right.
$$

We also require to define some compact notation to represent the multiple stochastic integral obtained from the Itô's Taylor expansion. So it is fundamental to define those integral on an appropriate set of integrable function. So let define:

$$
\begin{aligned}
\mathcal{H}_{(v)} & =\left\{g: \sup _{t \in[0, T]} E(|g(t)|)<\infty\right\} \\
\mathcal{H}_{(0)} & =\left\{g: E\left(\int_{0}^{T}|g(s)| d s\right)<\infty\right\} \\
\mathcal{H}_{(j)} & =\left\{g: E\left(\int_{0}^{T}|g(s)|^{2} d s\right)<\infty\right\},
\end{aligned}
$$

for $j \in\{1,2, \ldots m\}$, and $\mathcal{H}_{(\alpha)}$ define the set of all predictable process $g()$ for which the following multiple stochastic integral is defined.

$$
I_{\alpha}[g()]_{\rho, \tau}:=\left\{\begin{array}{lr}
g(\rho) & \text { if } l=0 \text { and } \alpha=v \\
\int_{\rho}^{\tau} I_{\alpha-}[g()]_{\rho, z} d z & \text { if } l \geq 1 \text { and } j_{l}=0 \\
\int_{\rho}^{\tau} I_{\alpha-}[g()]_{\rho, z} d W_{z}^{j_{l}} & \text { if } l \geq 1 \text { and } j_{l} \in\{1,2, \ldots m\}
\end{array}\right.
$$


where $\rho$ and $\tau$ are two stopping times with $0 \leq \rho \leq \tau \leq T$, a.s.

In the more explicit case i.e in dimension one where $m=1$ and $d=1$, we have:

$(13) \Longrightarrow \bar{L}^{(0)} f(t, x):=\frac{\partial}{\partial t} f(t, x)+\mu(t, x) \frac{\partial}{\partial x} f(t, x)+\frac{1}{2} \sigma^{2} \frac{\partial^{2}}{\partial x^{2}} f(t, x)$

and

$(14) \Longrightarrow \bar{L}^{(1)} f(t, x):=\sigma \frac{\partial}{\partial x} f(t, x), \quad \forall t \in[0, T]$ and $x \in \mathbb{R}$.

Then considering the function $f(t, x)=x$ we have:

$$
(15) \Longrightarrow \bar{f}_{\alpha}(t, x):=\left\{\begin{array}{llr}
x & \text { for } & l(\alpha)=0 \\
\mu(t, x) & \text { for } & l(\alpha)=1, j_{1}=0 \\
\sigma(t, x) & \text { for } & l(\alpha)=1, j_{1}=1 \\
\bar{L}^{\left(j_{1}\right)} \bar{f}_{-\alpha}(t, x) & \text { for } & l(\alpha) \geq 2, j_{1} \in\{0,1\}
\end{array}\right.
$$

and

$$
(17) \Longrightarrow I_{\alpha}[g()]_{\rho, \tau}:= \begin{cases}g(\rho) & \text { if } l=0 \text { and } \alpha=v \\ \int_{\rho}^{\tau} I_{\alpha-}[g()]_{\rho, z} d z & \text { if } l \geq 1 \text { and } j_{l}=0 \\ \int_{\rho}^{\tau} I_{\alpha-}[g()]_{\rho, z} d W_{z} & \text { if } l \geq 1 \text { and } j_{l}=1\end{cases}
$$

Let's define now the set for $\gamma=1$, the hierachical set

$$
\mathcal{A}_{\gamma}:=\left\{\alpha \in \mathcal{M}_{m}: l(\alpha)+n(\alpha) \leq 2 \gamma\right\},
$$

we also define the remainder set $\mathcal{B}\left(\mathcal{A}_{\gamma}\right)$ of $\mathcal{A}_{\gamma}$ by

$$
\mathcal{B}\left(\mathcal{A}_{\gamma}\right)=\left(\alpha \in \mathcal{M}_{m}-\mathcal{A},-\alpha \in \mathcal{A}\right)
$$

For illustration we found that: $\mathcal{A}_{\gamma}=\{v ;(0) ;(1) ;(1,1)\}$ and $\mathcal{B}\left(\mathcal{A}_{\gamma}\right)=\{(0,0) ;(0,1) ;(1,0)\}$.

Using (19) and (18) we can write $Y_{t_{i+1}-}$ and $X_{t_{i+1}-}$ as follows:

$$
\begin{gathered}
Y_{t_{i+1}-}=Y_{t_{i}}+\sum_{\alpha \in \mathcal{F}_{\gamma} \backslash v} I_{\alpha}\left[\bar{f}_{\alpha}\left(t_{i}, Y_{t_{i}}\right)\right]_{t_{i}, t_{i+1}}=\sum_{\alpha \in \mathcal{A}_{\gamma}} I_{\alpha}\left[\bar{f}_{\alpha}\left(t, Y_{t_{i}}\right)\right]_{t_{i}, t_{i+1}} \\
X_{t_{i+1}{ }^{-}}=X_{t_{i}}+\sum_{\alpha \in \mathcal{F}_{\gamma} \backslash v} I_{\alpha}\left[\bar{f}_{\alpha}\left(t_{i}, Y_{t_{i}}\right)\right]_{t_{i}, t_{i+1}}+\sum_{\alpha \in \mathcal{B}\left(\mathcal{A}_{\gamma}\right)} I_{\alpha}\left[\bar{f}_{\alpha}\left(t_{i}, X_{t_{i}}\right)\right]_{t_{i}, t_{i+1}}
\end{gathered}
$$

(20) and (21) are called Wagner-Platen expansion, see

Definition The scheme $Y^{\Delta}$ is said to converge strongly with order $\gamma>0$ at time $T$ to the solution $X$ of a given (SDE) if there exists a positive constant $C$ such that:

$$
\varepsilon(\Delta)=\sqrt{\mathbb{E}\left(\left|X_{T}-Y_{T}\right|^{2}\right)} \leq C \Delta^{\gamma}
$$

Assumption 1. For $\gamma=1$, let $Y^{\Delta}=\left\{Y_{t}, t \in[0, T]\right\}$ be the order $\gamma$ jump adapted time discretization with step size $\Delta$. Let consider the following assumptions:

$$
E\left(X_{0}\right) \leq \infty \text { and } E\left(\left|X_{0}-Y_{0}\right|^{2}\right) \leq K \Delta^{2 \gamma} .
$$

For $\alpha \in \mathcal{A}_{\gamma}, t \in[0, T]$ and $x, y \in \mathbb{R}^{d}$, the Itô coefficients satisfy the following Lipschitz condition

$$
\left|\bar{f}_{\alpha}(t, x)-\bar{f}_{\alpha}(t, y)\right| \leq K_{1}|x-y| .
$$

For all $\gamma \in \mathcal{A}_{\gamma} \cup \mathcal{B}\left(\mathcal{A}_{\gamma}\right)$

$$
\bar{f}_{-\alpha} \in C^{1,2} \text { and } \bar{f}_{\alpha} \in \mathcal{H}_{\alpha}
$$

for $t \in[0, T]$ and $x \in \mathbb{R}^{d}$

$$
\left|\bar{f}_{\alpha}(t, x)\right|^{2} \leq K_{2}\left(1+|x|^{2}\right)
$$


Theorem 1. Under the Assumptions(1), the following estimate

$$
\sqrt{\mathbb{E}\left(\sup _{0 \leq s \geq T}\left|X_{s}-Y_{s}\right|^{2} / \mathcal{A}_{0}\right)} \leq K_{3} \Delta^{\gamma}
$$

holds where the constant $K_{3}$ does not depend on $\Delta$

Before to prove the theorem above we have to get some preliminaries results. The following lemma is about the boundedness of the Itô multi-stochastic integral.

Lemma 1. For $\alpha \in \mathcal{M}_{m} \backslash\{v\}, g \in \mathcal{H}_{\alpha}$ and for two stopping times $\tau_{1}$ and $\tau_{2}$ such that $\mathcal{F}_{\tau_{1}}$-measurable and satisfying the conditions $t_{0} \leq \tau_{1} \leq \tau_{2} \leq \tau_{1}+\Delta \leq T$, a.s Then,

$$
\Phi_{\tau_{2}}^{\alpha} ;=E\left(\sup _{\tau_{1} \leq s \leq \tau_{2}}\left|I_{\alpha}[g(.)]_{\tau_{1}, s}\right|^{2} \mid \mathcal{F}_{\tau_{1}}\right) \leq 4^{l(\alpha)-n(\alpha)} \Delta^{l(\alpha)+n(\alpha)-1} \int_{\tau_{1}}^{\tau_{2}} P_{\tau_{1}, z}
$$

where

for $z \in\left[\tau_{1}, \tau_{2}\right]$

$$
P_{\tau_{1}, z}:=E\left(\sup _{\tau_{1} \leq t \leq z}|g(t)| \mid \mathcal{F}_{\tau_{1}}\right)<\infty
$$

Proof: The proof of (27) is done by induction on the different cases of $\alpha$, applying the Cauchy-Schwarz inequality we get: -When $\alpha=(0)$ with $l(\alpha)=1$ and $n(\alpha)=1$

$$
\left|\int_{\tau_{1}}^{s} g(z) d z\right|^{2} \leq\left(s-\tau_{1}\right) \int_{\tau_{1}}^{s}|g(z)|^{2} d z
$$

That leads to:

$$
\begin{gathered}
\Phi_{\tau_{2}}^{\alpha}=E\left(\sup _{\tau_{1} \leq s \leq \tau_{2}}\left|\int_{\tau_{1}}^{s} g(z) d z\right| \mid \mathcal{F}_{\tau_{1}}\right) \\
\leq E\left(\sup _{\tau_{1} \leq s \leq \tau_{2}}\left(s-\tau_{1}\right) \int_{\tau_{1}}^{s}|g(z)|^{2} d z \mid \mathcal{F}_{\tau_{1}}\right) \\
=E\left(\left(\tau_{2}-\tau_{1}\right) \int_{\tau_{1}}^{\tau_{2}}|g(z)|^{2} d z \mid \mathcal{F}_{\tau_{1}}\right) \\
\leq \Delta E\left(\int_{\tau_{1}}^{\tau_{2}}|g(z)|^{2} d z \mid \mathcal{F}_{\tau_{1}}\right) \\
\Phi_{\tau_{2}}^{\alpha} \leq \Delta \int_{\tau_{1}}^{\tau_{2}} E\left(|g(z)|^{2} \mid \mathcal{F}_{\tau_{1}}\right) d z \text { since } \tau_{2} \text { is } \mathcal{F}_{\tau_{1}}-\text { measurable } \\
=4^{l(\alpha)-n(\alpha)} \Delta^{l(\alpha)+n(\alpha)-1} \int_{\tau_{1}}^{\tau_{2}} P_{\tau_{1}, z},
\end{gathered}
$$

-When $\alpha=(1)$ with $l(\alpha)=1$ and $n(\alpha)=0$. The process

$$
\left\{I_{\alpha}[g(.)]_{\tau_{1}, t}, t \in\left[\tau_{1}, T\right]\right\}=\left\{\int_{\tau_{1}}^{t} g(s) d W_{s}, t \in\left[\tau_{1}, T\right]\right\}
$$

is a martingale. We have

$$
\begin{aligned}
\Phi_{\tau_{2}}^{\alpha} & =E\left(\sup _{\tau_{1} \leq s \leq \tau_{2}}\left|\int_{\tau_{1}}^{s} g(z) d W_{z}\right|^{2} \mid \mathcal{F}_{\tau_{1}}\right) \\
& \leq 4 E\left(\left|\int_{\tau_{1}}^{s} g(z) d W_{z}\right|^{2} \mid \mathcal{F}_{\tau_{1}}\right) \text { from the application of Doob's inequality } \\
& =4 E\left(\int_{\tau_{1}}^{\tau_{2}}|g(z)|^{2} d z \mid \mathcal{F}_{\tau_{1}}\right) \text { from the isometry formula }
\end{aligned}
$$


Since $\tau_{2}$ is $\mathcal{F}_{\tau_{1}}$-measurable we have

$$
\begin{aligned}
\Phi_{\tau_{2}}^{\alpha} & \leq E\left(\int_{\tau_{1}}^{\tau_{2}}|g(z)|^{2} d z \mid \mathcal{F}_{\tau_{1}}\right) \\
& =4 \int_{\tau_{1}}^{\tau_{2}} E\left(|g(t)|^{2} \mid \mathcal{F}_{\tau_{1}}\right) d z \\
& \leq 4 \int_{\tau_{1}}^{\tau_{2}} E\left(\sup _{\tau_{1} \leq t \leq z}|g(t)|^{2} \mid \mathcal{F}_{\tau_{1}}\right) d z \\
& =4^{l(\alpha)-n(\alpha)} \Delta^{l(\alpha)+n(\alpha)-1} \int_{\tau_{1}}^{\tau_{2}} P_{\tau_{1}, z},
\end{aligned}
$$

-When $\alpha=\left(j_{1}, j_{2}\right)$ with $j_{2}=0$ then $\forall j_{1}$ we have by applying the Cauchy-Schwarz inequality we get:

$$
\begin{aligned}
\Phi_{\tau_{2}}^{\alpha} & =E\left(\sup _{\tau_{1} \leq s \leq \tau_{2}}\left|\int_{\tau_{1}}^{s} I_{\alpha-}[g(.)]_{\tau_{1}, z} d z\right|^{2} \mid \mathcal{F}_{\tau_{1}}\right) \\
& \leq E\left(\sup _{\tau_{1} \leq s \leq \tau_{2}}\left(s-\tau_{1}\right) \int_{\tau_{1}}^{s}\left|I_{\alpha-}[g(.)]_{\tau_{1}, z}\right|^{2} d z \mid \mathcal{F}_{\tau_{1}}\right) \\
& =E\left(\left(\tau_{2}-\tau_{1}\right) \int_{\tau_{1}}^{\tau_{2}}\left|I_{\alpha-}[g(.)]_{\tau_{1}, z}\right|^{2} d z \mid \mathcal{F}_{\tau_{1}}\right) \\
& \leq \Delta E\left(\int_{\tau_{1}}^{\tau_{2}}\left|I_{\alpha-}[g(.)]_{\tau_{1}, z}\right|^{2} d z \mid \mathcal{F}_{\tau_{1}}\right) \\
& \leq \Delta E\left(\int_{\tau_{1}}^{\tau_{2}} \sup _{\tau_{1} \leq s \leq \tau_{2}}\left|I_{\alpha-}[g(.)]_{\tau_{1}, s}\right|^{2} d z \mid \mathcal{F}_{\tau_{1}}\right) \\
& =\Delta E\left(\int_{\tau_{1}}^{\tau_{2}} d z \times \sup _{\tau_{1} \leq s \leq \tau_{2}}\left|I_{\alpha-}[g(.)]_{\tau_{1}, s}\right|^{2} \mid \mathcal{F}_{\tau_{1}}\right) \\
& \leq \Delta^{2} E\left(\sup _{\tau_{1} \leq s \leq \tau_{2}}\left|I_{\alpha-}[g(.)]_{\tau_{1}, s}\right|^{2} \mid \mathcal{F}_{\tau_{1}}\right) \\
& =\Delta^{2} \Phi_{\tau_{2}}^{\alpha-}
\end{aligned}
$$

Considering the previous result we conclude that,

$$
\begin{aligned}
\Phi_{\tau_{2}}^{\alpha} & \leq \Delta^{2} 4^{l(\alpha-)-n(\alpha-)} \Delta^{l(\alpha-)+n(\alpha-)-1} \int_{\tau_{1}}^{\tau_{2}} P_{\tau_{1}, z} \\
& =4^{l(\alpha)-n(\alpha)} \Delta^{l(\alpha)+n(\alpha)-1} \int_{\tau_{1}}^{\tau_{2}} P_{\tau_{1}, z}
\end{aligned}
$$

Since $l(\alpha)=l(\alpha-)+1$ and $n(\alpha)=n(\alpha-)+1$

-When $\alpha=\left(j_{1}, j_{2}\right)$ with $j_{2}=1$ then $\forall j_{1}$, using the fact that the process

$$
\left\{I_{\alpha}[g(.)]_{\tau_{1}, t}, t \in\left[\tau_{1}, T\right]\right\}
$$

is a martingale we obtain through the application of Doob's inequality and Itô's isometry we get the expected result as 
follows

$$
\begin{aligned}
\Phi_{\tau_{2}}^{\alpha} & =E\left(\sup _{\tau_{1} \leq s \leq \tau_{2}}\left|\int_{\tau_{1}}^{s} I_{\alpha-}[g(.)]_{\tau_{1}, z} d W_{z}\right|^{2} \mid \mathcal{F}_{\tau_{1}}\right) \\
& \leq 4 E\left(\left|\int_{\tau_{1}}^{\tau_{2}} I_{\alpha-}[g(.)]_{\tau_{1}, z} d W_{z}\right|^{2} \mid \mathcal{F}_{\tau_{1}}\right) \text { Doob's inequality } \\
& =4 E\left(\int_{\tau_{1}}^{\tau_{2}}\left|I_{\alpha-}[g(.)]_{\tau_{1}, z}\right|^{2} d z \mid \mathcal{F}_{\tau_{1}}\right) \text { Itố's isometry } \\
& \leq 4\left(\int_{\tau_{1}}^{\tau_{2}} \sup _{\tau_{1} \leq s \leq \tau_{2}}\left|I_{\alpha-}[g(.)]_{\tau_{1}, s}\right|^{2} d z \mid \mathcal{F}_{\tau_{1}}\right) \\
& =4\left(\int_{\tau_{1}}^{\tau_{2}} d z \times \sup _{\tau_{1} \leq s \leq \tau_{2}}\left|I_{\alpha-}[g(.)]_{\tau_{1}, s}\right|^{2} \mid \mathcal{F}_{\tau_{1}}\right) \\
& =4\left(\left(\tau_{2}-\tau_{1}\right) \sup _{\tau_{1} \leq s \leq \tau_{2}}\left|I_{\alpha-}[g(.)]_{\tau_{1}, s}\right|^{2} \mid \mathcal{F}_{\tau_{1}}\right) \\
& \leq 4 \Delta\left(\sup _{\tau_{1} \leq s \leq \tau_{2}}\left|I_{\alpha-}[g(.)]_{\tau_{1}, s}\right|^{2} \mid \mathcal{F}_{\tau_{1}}\right) \\
& =4^{l(\alpha-)+1-n(\alpha-)} \Delta^{l(\alpha-)+1+n(\alpha-)-1} \int_{\tau_{1}}^{\tau_{2}} P_{\tau_{1}, z} \\
& =4^{l(\alpha)-n(\alpha)} \Delta^{l(\alpha)+n(\alpha)-1} \int_{\tau_{1}}^{\tau_{2}} P_{\tau_{1}, z}
\end{aligned}
$$

Since $l(\alpha)=l(\alpha-)+1$ and $n(\alpha)=n(\alpha-)$ and that completes the proof of the lemma(1).

Lemma 2. For $\alpha$ a given multi-index in $\mathcal{M}\{v\}$, a time discretization $\left\{t_{i}, i=0 \ldots N\right\}$ with step size $\Delta \in(0,1)$, and $g \in \mathcal{H}_{\alpha}$

$$
P_{t_{0}, u}:=E\left(\sup _{t_{0} \leq z \leq u}|g(z)|^{2} \mid \mathcal{F}_{t_{0}}\right)<\infty
$$

and

where

$$
\Phi_{t}^{\alpha}:=E\left(\sup _{t_{0} \leq z \leq t}\left|\sum_{i=0}^{i_{z}-1} I_{\alpha}[g(.)]_{t_{i}, t_{i+1}}+I_{\alpha}[g(.)]_{t_{z}, z}\right|^{2} \mid \mathcal{F}_{t_{0}}\right)
$$

$$
i_{t}=\max \left\{i \in\{0,1 \ldots\}: t_{i} \leq t\right\}
$$

Then

$$
\Phi_{t}^{\alpha}:=\left\{\begin{array}{lll}
\left(t-t_{0}\right) \Delta^{2(l(\alpha)-1)} \int_{t_{0}}^{t} P_{t_{0}, u} d u & \text { when : } l(\alpha)=n(\alpha) \\
4^{l(\alpha)-n(\alpha)+2} \Delta^{(l(\alpha)+n(\alpha)-1} \int_{t_{0}}^{t} P_{t_{0}, u} d u & \text { when: } l(\alpha) \neq n(\alpha)
\end{array}\right.
$$

Proof:

1. From the definition of $i_{z}$, for $z \in\left[t_{i}, t_{i+1}\right)$, the relation $t_{i_{z}}=t_{i}$ holds. Then, for a multi index $\alpha=\left(j_{1}, j_{2}\right)$ with $j_{2}=0$, we have

$$
\begin{aligned}
& \sum_{i=0}^{i_{z}-1} I_{\alpha}[g(.)]_{i_{i}, t_{i+1}}+I_{\alpha}[g(.)]_{t_{i}, z} \\
= & \sum_{i=0}^{i_{z}-1} \int_{t_{i}}^{t_{i+1}} I_{\alpha-}[g(.)]_{t_{i}, s} d s+\int_{t_{i_{z}}}^{z} I_{\alpha-}[g(.)]_{t_{i}, s} d s \\
= & \sum_{i=0}^{z_{z}-1} \int_{t_{i}}^{t_{i+1}} I_{\alpha-}[g(.)]_{t_{i}, s} d s+\int_{t_{i_{z}}}^{z} I_{\alpha-}[g(.)]_{i_{s}, s} d s \\
= & \int_{t_{0}}^{z} I_{\alpha-[}[g(.)]_{i_{s}, s} d s
\end{aligned}
$$


For $\alpha=\left(j_{1}, j_{2}\right)$ with $j_{2}=1$, we have

$$
\begin{aligned}
& \sum_{i=0}^{i_{z}-1} I_{\alpha}[g(.)]_{t_{i}, t_{i+1}}+I_{\alpha}[g(.)]_{t_{i}, z} \\
= & \sum_{i=0}^{i_{z}-1} \int_{t_{i}}^{t_{i+1}} I_{\alpha-}[g(.)]_{t_{i}, s} d W_{s}+\int_{t_{i_{z}}}^{z} I_{\alpha-}[g(.)]_{t_{z}, s} d W_{s} \\
= & \sum_{i=0}^{i_{z}-1} \int_{t_{i}}^{t_{i+1}} I_{\alpha-}[g(.)]_{t_{s}, s} d W_{s}+\int_{t_{i_{z}}}^{z} I_{\alpha-}[g(.)]_{t_{i}, s} d W_{s} \\
= & \int_{t_{0}}^{z} I_{\alpha-}[g(.)]_{t_{i}, s} d W_{s}
\end{aligned}
$$

2. Let consider the case $l(\alpha)=n(\alpha)$

$$
\begin{aligned}
& \Phi_{t}^{\alpha}=E\left(\left.\sup _{t_{0} \leq z \leq t}\left|\int_{t_{0}}^{z} I_{\alpha-}[g(.)]_{t_{i}, s} d s\right|\right|^{2} \mid \mathcal{F}_{t_{0}}\right) \\
& \leq E\left(\sup _{t_{0} \leq z \leq t}\left(z-t_{0}\right) \int_{t_{0}}^{z}\left|I_{\alpha-}[g(.)]_{t_{s}, s}\right|^{2} d s \mid \mathcal{F}_{t_{0}}\right) \text { Cauchy-Schwarz inequality } \\
& \leq\left(t-t_{0}\right) E\left(\int_{t_{0}}^{t}\left|I_{\alpha-}[g(.)]_{t_{s}, s}\right|^{2} d s \mid \mathcal{F}_{t_{0}}\right) \\
& =\left(t-t_{0}\right) \int_{t_{0}}^{t} E\left(\left|I_{\alpha-}[g(.)]_{t_{s}, s}\right|^{2} \mid \mathcal{F}_{t_{0}}\right) d s \\
& \leq\left(t-t_{0}\right) \int_{t_{0}}^{t} E\left(\sup _{t_{i s} \leq z \leq s}\left|I_{\alpha-}[g(.)]_{t_{i}, z}\right|^{2} \mid \mathcal{F}_{t_{0}}\right) d s \\
& =\left(t-t_{0}\right) \int_{t_{0}}^{t} E[\underbrace{E\left(\sup _{t_{s} \leq z \leq s}\left|I_{\alpha-}[g(.)]_{i_{s}, z}\right|^{2} \mid \mathcal{F}_{t_{s}}\right)}_{=\Phi_{s}^{\alpha-}} \mid \mathcal{F}_{t_{0}}] d s
\end{aligned}
$$

Since $t_{0} \leq t_{i_{s}}$ and then $\mathcal{F}_{t_{0}} \subseteq \mathcal{F}_{t_{i s}}$ for $s \in\left[t_{0}, t\right]$.

From Lemma(1) we subtitute the value of $\Phi_{s}^{\alpha-}$ into (44) and yields

$$
\begin{aligned}
\Phi_{t}^{\alpha} \leq & \left(t-t_{0}\right) 4^{l(\alpha-)-n(\alpha-)} \\
& \times \int_{t_{0}}^{t} E\left(\Delta^{l(\alpha-)+n(\alpha-)-1} \int_{t_{i s}}^{s} P_{t_{s}, z} d z \mid \mathcal{F}_{t_{0}}\right) d s \\
\leq & \left(t-t_{0}\right) 4^{l(\alpha-)-n(\alpha-)} \int_{t_{0}}^{t} E\left(\Delta^{l(\alpha-)+n(\alpha-)-1}\left(s-t_{i_{s}}\right) P_{t_{t_{s}}, s} \mid \mathcal{F}_{t_{0}}\right) d s \\
\leq & \left(t-t_{0}\right) 4^{l(\alpha-)-n(\alpha-)} \Delta^{l(\alpha-)+n(\alpha-)} \int_{t_{0}}^{t} E\left(P_{t_{i_{s}}, s} \mid \mathcal{F}_{t_{0}}\right) d s
\end{aligned}
$$

since $\left(s-t_{i_{s}}\right) \leq \Delta$. Given that $\mathcal{F}_{t_{0}} \subseteq \mathcal{F}_{t_{i s}}$ for $s \in\left[t_{0}, t\right]$

$$
\begin{aligned}
E\left(P_{t_{i s}, s} \mid \mathcal{F}_{t_{0}}\right) & =E\left(E\left(\sup _{t_{i s} \leq u \leq s}|g(u)|^{2} \mid \mathcal{F}_{t_{i_{s}}}\right) \mid \mathcal{F}_{t_{0}}\right) \\
& =E\left(\sup _{t_{i_{s} \leq u \leq s}}|g(u)|^{2} \mid \mathcal{F}_{t_{0}}\right) \\
& \leq E\left(\sup _{t_{0} \leq u \leq s}|g(u)|^{2} \mid \mathcal{F}_{t_{0}}\right) \\
& =P_{t_{0}, s}
\end{aligned}
$$


It then follows

$$
\begin{aligned}
\Phi_{t}^{\alpha} & \leq\left(t-t_{0}\right) 4^{l(\alpha-)-n(\alpha-)} \Delta^{l(\alpha-)+n(\alpha-)} \int_{t_{0}}^{t} P_{t_{0}, s} d s \\
& =\left(t-t_{0}\right) \Delta^{2 l(l \alpha)-1)}
\end{aligned}
$$

That completes then the proof of the case $l(\alpha)=n(\alpha)$.

3. Let consider the case for the multi-index $\alpha=\left(j_{1}, j_{2}\right)$ such that $l(\alpha) \neq n(\alpha)$ and $j_{2}=1$. For that we know that the multiple stochastic integral $\left\{\int_{t_{0}}^{z} I_{\alpha-}[g(.)]_{t_{s}, s} d W_{s}\right\}$ is a martingale. We then have

$$
\begin{aligned}
\Phi_{t}^{\alpha}= & E\left(\sup _{t_{0} \leq z \leq t}\left|\int_{t_{0}}^{z} I_{\alpha-}[g(.)]_{t_{s}, s} d W_{s}\right|^{2} \mid \mathcal{F}_{t_{0}}\right) \\
\leq & 4 E\left(\left|\int_{t_{0}}^{z} I_{\alpha-}[g(.)]_{t_{s}, s} d W_{s}\right|^{2} \mid \mathcal{F}_{t_{0}}\right) \text { Doob's inequality } \\
= & 4 E\left(\int_{t_{0}}^{z}\left|I_{\alpha-}[g(.)]_{t_{s}, s}\right|^{2} d s \mid \mathcal{F}_{t_{0}}\right) \text { Itô's isometry } \\
\leq & \int_{t_{0}}^{z} E\left(\left|I_{\alpha-}[g(.)]_{t_{i s}, s}\right|^{2} \mid \mathcal{F}_{t_{0}}\right) d s \\
\Phi_{t}^{\alpha} & \leq \int_{t_{0}}^{z} E\left(E\left(\left|I_{\alpha-}[g(.)]_{t_{i s}, s}\right|^{2} \mid \mathcal{F}_{t_{t_{s}}}\right) \mid \mathcal{F}_{t_{0}}\right) d s \\
\leq & 4 E \underbrace{E\left(\sup _{t_{i s} \leq z \leq s}\left|I_{\alpha-}[g(.)]_{t_{s}, z}\right|^{2} \mid \mathcal{F}_{t_{i_{s}}}\right)}_{=\Phi_{s}^{\alpha-}}\left|\mathcal{F}_{t_{0}}\right| d s \\
\leq & 44^{l(\alpha-)-n(\alpha-)} \mid \\
& \times \int_{t_{0}}^{t} E\left(\Delta^{l(\alpha-)+n(\alpha-)-1} \int_{t_{i_{s}}}^{s} P_{t_{t_{s}}, z} d z \mid \mathcal{F}_{t_{0}}\right) d s \\
\leq & 4^{l(\alpha-)-n(\alpha-)+1} \Delta^{l(\alpha-)+n(\alpha-)} \int_{t_{0}}^{t} E\left(P_{t_{i_{s}}, s} \mid \mathcal{F}_{t_{0}}\right) d s
\end{aligned}
$$

And as the previous proof it leads to

$$
\begin{aligned}
\Phi_{t}^{\alpha} & \leq 4^{l(\alpha-)-n(\alpha-)+1} \Delta^{l(\alpha-)+n(\alpha-)} \int_{t_{0}}^{t} P_{t_{0}, s} d s \\
& =4^{l(\alpha)-n(\alpha)} \Delta^{l(\alpha)+n(\alpha)-1} \int_{t_{0}}^{t} P_{t_{0}, s} d s
\end{aligned}
$$

since $l(\alpha-)=l(\alpha)-1$ and $n(\alpha-)=n(\alpha)$.

4 To complete the proof we finally consider the case of $\alpha=\left(j_{1}, j_{2}\right)$ such that $l(\alpha) \neq n(\alpha)$ and $j_{2}=0$. Applying Cauchy Schwarz inequality on $\Phi_{t}^{\alpha}$, we get

$$
\begin{aligned}
\Phi_{t}^{\alpha}= & E\left(\left.\sup _{t_{0} \leq z \leq t}\left|\sum_{i=0}^{i_{z}-1} I_{\alpha}[g(.)]_{t_{i}, t_{i+1}}+I_{\alpha}[g(.)]_{i_{z}, z}\right|\right|^{2} \mid \mathcal{F}_{t_{0}}\right) \\
\leq & 2 E\left(\left.\sup _{t_{0} \leq z \leq t}\left|\sum_{i=0}^{i_{z}-1} I_{\alpha}[g(.)]_{t_{i}, t_{i+1}}\right|\right|^{2} \mid \mathcal{F}_{t_{0}}\right) \\
& +2 E\left(\sup _{t_{0} \leq z \leq t}\left|I_{\alpha}[g(.)]_{t_{i}, z}\right|^{2} \mid \mathcal{F}_{t_{0}}\right)
\end{aligned}
$$


$\sum_{i=0}^{n} I_{\alpha}[g(.)]_{i, t_{i+1}}+I_{\alpha}[g(.)]_{t_{i}, z}$ is a discrete time martingale, see (Kloeden \& Platen, 1992) Then considering the first term of (51) we have,

$$
\begin{aligned}
& E\left(\sup _{t_{0} \leq z \leq t}\left|\sum_{i=0}^{i_{z}-1} I_{\alpha}[g(.)]_{t_{i}, t_{i+1}}\right|^{2} \mid \mathcal{F}_{t_{0}}\right) \\
& \leq 4 E\left(\left|\sum_{i=0}^{i_{t}-1} I_{\alpha}[g(.)]_{t_{i}, t_{i+1}}\right|^{2} \mid \mathcal{F}_{t_{0}}\right) \text { Doob's Inequality } \\
& =4 E\left(\left|\sum_{i=0}^{i_{t}-2} I_{\alpha}[g(.)]_{i_{i}, t_{i+1}}+I_{\alpha}[g(.)]_{t_{i-1}, t_{t_{i}}}\right|^{2} \mid \mathcal{F}_{t_{0}}\right) \\
& \leq 4 E\left(\left[\left|\sum_{i=0}^{i_{t}-2} I_{\alpha}[g(.)]_{i_{i}, t_{i+1}}\right|^{2}+2\left|\sum_{i=0}^{i_{t}-2} I_{\alpha}[g(.)]_{t_{i}, t_{i+1}}\right| E\left(\left|I_{\alpha}[g(.)]_{t_{i-1}, t_{i t}}\right| \mid \mathcal{F}_{t_{i t}-1}\right)\right.\right. \\
& +E\left(\left|I_{\alpha}[g(.)]_{t_{t_{t}-1}, t_{i}}\right|^{2}\left|\mathcal{F}_{t_{i_{t}}-1}\right| \mathcal{F}_{t_{0}}\right) \\
& \leq 4 E\left(\left[\left|\sum_{i=0}^{i_{t}-2} I_{\alpha}[g(.)]_{i_{i}, t_{i+1}}\right|^{2}\right.\right. \\
& \left.+E\left(\left|I_{\alpha}[g(.)]_{t_{i-1}, t_{i}}\right|^{2} \mid \mathcal{F}_{t_{t_{t}}-1}\right) \mid \mathcal{F}_{t_{0}}\right)
\end{aligned}
$$

since from the discrete martingale property of the stochastic integral $I_{\alpha}[g(.)]_{i_{i-1}, t_{i_{t}}}$ we have $E\left(I_{\alpha}[g(.)]_{i_{i-1}, t_{i t}} \mid \mathcal{F}_{t_{i_{i}}-1}=0\right.$. By applying the Lemma(1) on $E\left(\left|I_{\alpha}[g(.)]_{t_{i-1}, t_{t_{i}}}\right|^{2} \mid \mathcal{F}_{t_{i_{t}}-1}\right)$ we get

$$
\begin{aligned}
& E\left(\left.\sup _{t_{0} \leq z \leq t}\left|\sum_{i=0}^{i_{z}-1} I_{\alpha}[g(.)]_{t_{i}, t_{i+1}}\right|\right|^{2} \mid \mathcal{F}_{t_{0}}\right) \\
\leq & 4 E\left(\left[\left|\sum_{i=0}^{i_{t}-2} I_{\alpha}[g(.)]_{i_{i}, t_{i+1}}\right|^{2}\right.\right. \\
& \left.\left.+4^{l(\alpha)-n(\alpha)} \Delta^{l(\alpha)+n(\alpha)-1} \int_{t_{t_{i}-1}}^{t_{i_{t}}} P_{t_{t-1}, u} d u\right] \mid \mathcal{F}_{t_{0}}\right)
\end{aligned}
$$


Expanding $\sum_{i=0}^{i_{t}-2} I_{\alpha}[g(.)]_{t_{i}, t_{i+1}}$ as $\sum_{i=0}^{i_{t}-1} I_{\alpha}[g(.)]_{t_{i}, t_{i+1}}$ we obtain

$$
\begin{aligned}
& E\left(\left.\sup _{t_{0} \leq z \leq t}\left|\sum_{i=0}^{i_{z}-1} I_{\alpha}[g(.)]_{t_{i}, t_{i+1}}\right|\right|^{2} \mathcal{F}_{t_{0}}\right) \\
& \leq 4 E\left(\left[\left|\sum_{i=0}^{i_{t}-3} I_{\alpha}[g(.)]_{t_{i}, t_{i+1}}\right|^{2}\right.\right. \\
& +4^{l(\alpha)-n(\alpha)} \Delta^{l(\alpha)+n(\alpha)-1} \int_{t_{i_{t}-2}}^{t_{t_{t}}-1} P_{t_{i_{t}-2}, u} d u \\
& \left.+4^{l(\alpha)-n(\alpha)} \Delta^{l(\alpha)+n(\alpha)-1} \int_{t_{t_{t}-1}}^{t_{i_{t}}} P_{t_{t_{t}-1}, u} d u \| \mathcal{F}_{t_{0}}\right) \\
& \leq 4 E\left(\left[\left|\sum_{i=0}^{i_{t}-3} I_{\alpha}[g(.)]_{t_{i}, t_{i+1}}\right|^{2}\right.\right. \\
& +4^{l(\alpha)-n(\alpha)} \Delta^{l(\alpha)+n(\alpha)-1} \int_{t_{i_{t}-2}}^{t_{t_{t}}-1} P_{t_{t_{t}-2}, u} d u \\
& \left.\left.+4^{l(\alpha)-n(\alpha)} \Delta^{l(\alpha)+n(\alpha)-1} \int_{t_{i_{t}-1}}^{t_{i_{t}}} P_{t_{t_{t}-2}, u} d u\right] \mid \mathcal{F}_{t_{0}}\right) \text { since } P_{t_{i_{t}-1}, u} \leq P_{t_{t_{t}-2}, u} \\
& \leq 4 E\left(\left[\left|\sum_{i=0}^{i_{t}-3} I_{\alpha}[g(.)]_{t_{i}, t_{i+1}}\right|^{2}\right.\right. \\
& \left.+4^{l(\alpha)-n(\alpha)} \Delta^{l(\alpha)+n(\alpha)-1} \int_{t_{t_{t}-2}}^{t_{i_{t}}} P_{t_{t_{t}-2}, u} d u \| \mathcal{F}_{t_{0}}\right)
\end{aligned}
$$

and repetitively it leads to

$$
\begin{aligned}
E\left(\sup _{t_{0} \leq z \leq t}\left|\sum_{i=0}^{i_{z}-1} I_{\alpha}[g(.)]_{t_{i}, t_{i+1}}\right|^{2} \mid \mathcal{F}_{t_{0}}\right) & \leq 4^{l(\alpha)-n(\alpha)+1} \Delta^{l(\alpha)+n(\alpha)-1} E\left(\int_{t_{0}}^{t} P_{t_{0}, u} d u \mid \mathcal{F}_{t_{0}}\right) \\
& =4^{l(\alpha)-n(\alpha)+1} \Delta^{l(\alpha)+n(\alpha)-1} \int_{t_{0}}^{t} P_{t_{0}, u} d u
\end{aligned}
$$

Considering now the secoond term of equation (51) using Cauchy Schwarz inequality we have

$$
\begin{aligned}
E\left(\sup _{t_{0} \leq z \leq t}\left|I_{\alpha}[g(.)]_{t_{i z}, z}\right|^{2} \mid \mathcal{F}_{t_{0}}\right) & =E\left(\sup _{t_{0} \leq z \leq t}\left|\int_{t_{i_{z}}}^{z} I_{\alpha}[g(.)]_{t_{z}, u} d u\right|^{2} \mid \mathcal{F}_{t_{0}}\right) \\
& \leq E\left(\sup _{t_{0} \leq z \leq t}\left(z-t_{i_{z}}\right) \int_{t_{i_{z}}}^{z}\left|I_{\alpha}[g(.)]_{t_{z}, u}\right|^{2} d u \mid \mathcal{F}_{t_{0}}\right)
\end{aligned}
$$

Similarly as in the proof of Lemma(1) we obtain

$$
E\left(\sup _{t_{0} \leq z \leq t}\left|I_{\alpha}[g(.)]_{i_{z}, z}\right|^{2} \mid \mathcal{F}_{t_{0}}\right) \leq 4^{l(\alpha)-n(\alpha)} \Delta^{l(\alpha)+n(\alpha)-1} \int_{t_{0}}^{t} P_{t_{0}, u} d u .
$$

Therefore, from the equations (54) and (54) we finally get

$$
\begin{aligned}
\Phi_{t}^{\alpha} & \leq 2\left(4^{l(\alpha)-n(\alpha)+1} \Delta^{l(\alpha)+n(\alpha)-1} \int_{t_{0}}^{t} P_{t_{0}, u} d u+4^{l(\alpha)-n(\alpha)} \Delta^{l(\alpha)+n(\alpha)-1} \int_{t_{0}}^{t} P_{t_{0}, u} d u\right) \\
& \leq 4^{l(\alpha)-n(\alpha)+2} \Delta^{l(\alpha)+n(\alpha)-1} \int_{t_{0}}^{t} P_{t_{0}, u} d u,
\end{aligned}
$$

and that completes the proof of Lemma(2).

The following Lemma is an intermediary result required to handle the moment of the solution of the SDE(2). 
Lemma 3. Assuming conditions (3) and (4) satisfied and let

$$
E\left(\left|X_{t_{0}}\right|^{2}\right)<\infty
$$

Then the solution $X_{t}$ of (2) satisfies

$$
E\left(\sup _{t_{0} \leq s \leq T}\left|X_{s}\right|^{2} \mid \mathcal{F}_{t_{0}}\right) \leq C\left(1+E\left(\left|X_{t_{0}}\right|^{2}\right)\right)
$$

for every $t \in\left[t_{0}, T\right]$, with $C$ a positive constant that depends only on $\left(T-t_{0}\right)$ and the linear growth bound.

Proof of Theorem(1)

Considering the Wagner-Platen expansion for the diffusion process as described in equations(20) and (21) the solution of the $\operatorname{SDE}(2)$ at time $t \in[0, T]$ can be written as follows:

$$
\begin{aligned}
X_{t}= & X_{0}+\sum_{\alpha \in \mathcal{A}_{\gamma} \backslash v}\left\{\sum_{i=o}^{i_{t}-1} I_{\alpha}\left[\bar{f}_{\alpha}\left(t_{i}, X_{t_{i}}\right)\right]_{t_{i}, t_{i+1}}+I_{\alpha}\left[\bar{f}_{\alpha}\left(t_{i_{t}}, X_{t_{i}}\right)\right]_{t_{t}, t}\right\} \\
& +\sum_{\alpha \in \mathcal{B}\left(\mathcal{A}_{\gamma}\right)}\left\{\sum_{i=o}^{i_{t}-1} I_{\alpha}\left[\bar{f}_{\alpha}\left(t_{i}, X_{t_{i}}\right)\right]_{t_{i}, t_{i+1}}+I_{\alpha}\left[\bar{f}_{\alpha}\left(t_{t_{t}}, X_{t_{i}}\right)\right]_{t_{t}, t}\right\} \\
& +\int_{0}^{t} J\left(X_{t_{i_{s}}}\right) d N_{s},
\end{aligned}
$$

and the jump adapted scheme is

$$
Y_{t}=Y_{0}+\sum_{\alpha \in \mathcal{A}_{\gamma} \backslash v}\left\{\sum_{i=o}^{i_{t}-1} I_{\alpha}\left[\bar{f}_{\alpha}\left(t_{i}, Y_{t_{i}}\right)\right]_{t_{i}, t_{i+1}}+I_{\alpha}\left[\bar{f}_{\alpha}\left(t_{i_{t}}, Y_{t_{i}}\right)\right]_{i_{t}, t}\right\}+\int_{0}^{t} J\left(Y_{t_{i_{s}}}\right) d N_{s}
$$

for $t \in[0, T]$. From Lemma(3) we have

$$
E\left(\sup _{t_{0} \leq s \leq T}\left|X_{s}\right|^{2} \mid \mathcal{F}_{t_{0}}\right) \leq C\left(1+E\left(\left|X_{t_{0}}\right|^{2}\right)\right)
$$

Now let's prove the same result for the jump adapted scheme.

$$
\begin{aligned}
& E\left(\sup _{0 \leq s \leq T}\left|Y_{s}\right|^{2} \mid \mathcal{F}_{t_{0}}\right) \leq E\left(\sup _{0 \leq s \leq T}\left(1+\left|Y_{s}\right|^{2}\right) \mid \mathcal{F}_{0}\right) \\
& \leq E\left(\operatorname { s u p } _ { 0 \leq s \leq T } \left(1+\mid Y_{0}+\sum_{\alpha \in \mathcal{A}_{\gamma} \backslash v}\left\{\sum_{i=o}^{i_{t}-1} I_{\alpha}\left[\bar{f}_{\alpha}\left(t_{i}, Y_{t_{i}}\right)\right]_{t_{i}, t_{i+1}}\right.\right.\right. \\
& \left.\left.+I_{\alpha}\left[\bar{f}_{\alpha}\left(t_{i_{t}}, Y_{t_{i}}\right)\right]_{t_{i_{t}}, t}+\left.\int_{0}^{s} J\left(X_{t_{u_{u}}}-\right) d N_{u}\right|^{2}\right) \mid \mathcal{F}_{t_{0}}\right) \\
& \leq E\left(\operatorname { s u p } _ { 0 \leq s \leq T } \left(1+2\left|Y_{0}\right|^{2}+4 \mid \sum_{\alpha \in \mathcal{A}_{\gamma} \backslash v}\left\{\sum_{i=o}^{i_{t}-1} I_{\alpha}\left[\bar{f}_{\alpha}\left(t_{i}, Y_{t_{i}}\right)\right]_{t_{i}, t_{i+1}}\right.\right.\right. \\
& \left.\left.+\left.I_{\alpha}\left[\bar{f}_{\alpha}\left(t_{i_{t}}, Y_{t_{i}}\right)\right]_{i_{t}, t}\right|^{2}+4\left|\int_{0}^{s} J\left(X_{t_{i_{u}}-}\right) d N_{u}\right|^{2}\right) \mid \mathcal{F}_{0}\right) \\
& \leq E\left(\sup _{0 \leq s \leq T}\left(1+\left|Y_{0}\right|^{2}\right)\right)+4 \sum_{\alpha \in \mathcal{A}_{\gamma} \backslash\{v\}} E\left(\sup _{0 \leq s \leq T} \mid \sum_{i=o}^{i_{t}-1} I_{\alpha}\left[\bar{f}_{\alpha}\left(t_{i}, Y_{t_{i}}\right)\right]_{t_{i}, t_{i+1}}\right. \\
& \left.+\left.I_{\alpha}\left[\bar{f}_{\alpha}\left(t_{i_{t}}, Y_{t_{i}}\right)\right]_{t_{t}, t}\right|^{2} \mid \mathcal{F}_{0}\right)+4 E\left(\sup _{0 \leq s \leq T}\left|\int_{0}^{s} J\left(X_{t_{i_{u}}}\right) d N_{u}\right|^{2} \mid \mathcal{F}_{0}\right)
\end{aligned}
$$


Then from the Lema(2) and the Linear growth condition(25) we have

$$
\begin{aligned}
& \sum_{\alpha \in \mathcal{A}_{\gamma} \backslash\{v\}} E\left(\sup _{0 \leq s \leq T}\left|\sum_{i=o}^{i_{t}-1} I_{\alpha}\left[\bar{f}_{\alpha}\left(t_{i}, Y_{t_{i}}\right)\right]_{t_{i}, t_{i+1}}+I_{\alpha}\left[\bar{f}_{\alpha}\left(t_{i_{t}}, Y_{t_{i}}\right)\right]_{t_{i_{t}}, t}\right|^{2} \mid \mathcal{F}_{0}\right) \\
& \leq K_{2} \sum_{\alpha \in \mathcal{F}_{\gamma} \backslash\{v\}} \int_{0}^{T} E\left(\sup _{t_{0} \leq s \leq u}\left|\bar{f}_{\alpha}\left(s, Y_{s}\right)\right|^{2} \mid \mathcal{F}_{0}\right) d u \\
& \leq K_{2} \sum_{\alpha \in \mathcal{A}_{\gamma} \backslash\{v\}} \int_{0}^{T} E\left(\sup _{t_{0} \leq s \leq u}\left(1+\left|Y_{s}\right|^{2}\right) \mid \mathcal{F}_{0}\right) d u \\
& \leq C_{2}^{\prime} \int_{0}^{T} E\left(\sup _{t_{0} \leq s \leq u}\left(1+\left|Y_{s}\right|^{2}\right) \mid \mathcal{F}_{0}\right) d u \\
& E\left(\sup _{0 \leq s \leq T}\left|\int_{0}^{s} J\left(X_{t_{i_{u}}-}\right) d N_{u}\right|^{2} \mid \mathcal{F}_{0}\right) \\
& \leq E\left(\sup _{t_{0} \leq s \leq T}\left|\int_{0}^{s} J\left(X_{t_{i_{u}}-}\right) d \tilde{N}_{u}+\lambda \int_{0}^{s} J\left(X_{t_{i_{u}}-}\right) d u\right|^{2} \mid \mathcal{F}_{0}\right), \quad \begin{array}{l}
\text { where } \tilde{N} \text { is the compensated } \\
\text { poisson measure }
\end{array} \\
& \leq 2 E\left(\sup _{t_{0} \leq s \leq T}\left|\int_{0}^{s} J\left(X_{t_{i_{u}}}\right) d \tilde{N}_{u}\right|^{2} \mid \mathcal{F}_{0}\right)+2 E\left(\sup _{t_{0} \leq s \leq T}\left|\int_{0}^{s} J\left(X_{t_{i_{u}}-}\right) d u\right|^{2} \mid \mathcal{F}_{0}\right) \\
& \leq 8 E\left(\mid \int_{0}^{T} J\left(\left.X_{t_{i_{u}}} d \tilde{N}_{u}\right|^{2} \mid \mathcal{F}_{0}\right)+2 E\left(\sup _{t_{0} \leq s \leq T}\left|\int_{0}^{s} J\left(X_{t_{i_{u}}}\right) d u\right|^{2} \mid \mathcal{F}_{0}\right)\right.
\end{aligned}
$$

Using the Itô's isometry for jump and the linear growth condition (4) we get

$$
E\left(\sup _{0 \leq s \leq T}\left|\int_{0}^{s} J\left(X_{t_{i u}-}\right) d N_{u}\right|^{2} \mid \mathcal{F}_{0}\right) \leq C_{3}^{\prime} \int_{0}^{T} E\left(\sup _{t_{0} \leq s \leq u}\left(1+\left|Y_{s}\right|^{2}\right) \mid \mathcal{F}_{0}\right) d u
$$

Subtituting (59) and (60) into (59) we have

$$
E\left(\sup _{0 \leq s \leq T}\left|Y_{s}\right|^{2} \mid \mathcal{F}_{0}\right) \leq C_{1}^{\prime}\left(1+E\left(\left|Y_{0}\right|^{2}\right)\right)+C_{4}^{\prime} \int_{0}^{T} E\left(\sup _{t_{0} \leq s \leq u}\left(1+\left|Y_{s}\right|^{2}\right) \mid \mathcal{F}_{0}\right) d u
$$

Then by applying the Gronwall inequality, we obtain

$$
E\left(\sup _{0 \leq s \leq T}\left|Y_{s}\right|^{2} \mid \mathcal{F}_{0}\right) \leq C^{\prime}\left(1+E\left(\left|Y_{0}\right|^{2}\right)\right)
$$

where $C^{\prime}$ is a positive constant. The final step of proof consists of analyzing the mean square given by

$$
\begin{aligned}
Z(t):= & E\left(\sup _{0 \leq s \leq t}\left|X_{s}-Y_{s}\right|^{2} \mid \mathcal{F}_{0}\right) \\
\leq & E\left(\sup _{0 \leq s \leq t} \mid X_{0}-Y_{0}+\sum_{\alpha \in \mathcal{F}_{\gamma} \backslash\{v\}}\left\{\sum_{i=0}^{i_{s}-1} I_{\alpha}\left[\bar{f}_{\alpha}\left(t_{i}, X_{t_{i}}\right)-\bar{f}_{\alpha}\left(t_{i}, Y_{t_{i}}\right)\right]_{t_{i}, t_{i+1}}\right.\right. \\
& +I_{\alpha}\left[\bar{f}_{\alpha}\left(t_{i_{s}}, X_{t_{i s}}\right)-\bar{f}_{\alpha}\left(t_{i_{s}}, Y_{t_{i s}}\right)\right]_{t_{i s}, s} \\
& +\sum_{\alpha \in \mathcal{B}\left(\mathcal{A}_{\gamma}\right)}\left\{\sum_{i=o}^{i_{t}-1} I_{\alpha}\left[\bar{f}_{\alpha}\left(t_{i}, X_{t_{i}}\right)\right]_{t_{i}, t_{i+1}}+I_{\alpha}\left[\bar{f}_{\alpha}\left(t_{i_{s}}, X_{t_{i_{s}}}\right)\right]_{t_{i_{s}}, s}\right\} \\
& \left.+\left.\int_{0}^{s}\left\{J\left(X_{t_{i_{u}}-}\right)-J\left(Y_{t_{i_{u}}-}\right)\right\} d N_{u}\right|^{2} \mid \mathcal{F}_{0}\right) \\
\leq & C s t e\left\{\left|x_{0}-Y_{0}\right|^{2}+\sum_{\alpha \in \mathcal{F}_{\gamma} \backslash\{v\}} A_{t}^{\alpha}+\sum_{\alpha \in \mathcal{B}\left(\mathcal{A}_{\gamma}\right)} B_{t}^{\alpha}+D_{t}\right\}
\end{aligned}
$$


$\forall t \in[0, T]$ where $A_{t}^{\alpha}, B_{t}^{\alpha}$ and $D_{t}$ are defined as follows

$$
\begin{aligned}
& A_{t}^{\alpha}:= E\left(\sup _{0 \leq s \leq t} \mid \sum_{i=0}^{i_{s}-1} I_{\alpha}\left[\bar{f}_{\alpha}\left(t_{i}, X_{t_{i}}\right)-\bar{f}_{\alpha}\left(t_{i}, Y_{t_{i}}\right)\right]_{i_{i}, t_{i+1}}\right. \\
&\left.\left.+I_{\alpha}\left[\bar{f}_{\alpha}\left(t_{i_{s}}, X_{t_{i s}}\right)-\bar{f}_{\alpha}\left(t_{i_{s}}, Y_{t_{s}}\right)\right]\right]_{t_{s}, s},\left.\right|^{2} \mid \mathcal{F}_{0}\right), \\
& B_{t}^{\alpha}:=E\left(\sup _{0 \leq s \leq t}\left|\sum_{i=o}^{i_{t}-1} I_{\alpha}\left[\bar{f}_{\alpha}\left(t_{i}, X_{t_{i}}\right)\right]_{t_{i}, t_{i+1}}+I_{\alpha}\left[\bar{f}_{\alpha}\left(t_{i_{s}}, X_{t_{i s}}\right)\right]_{t_{i s}, s}\right|^{2} \mid \mathcal{F}_{0}\right),
\end{aligned}
$$

and

$$
D_{t}:=E\left(\sup _{0 \leq s \leq t}\left|\int_{0}^{s}\left\{J\left(X_{t_{i_{u}}}\right)-J\left(Y_{t_{i_{u}}}\right)\right\} d N_{u}\right|^{2} \mid \mathcal{F}_{0}\right)
$$

Now by using the Lemma(2)and Lipschitz(23) we get

$$
\begin{aligned}
A_{t}^{\alpha}:= & E\left(\sup _{0 \leq s \leq t} \mid \sum_{i=0}^{i_{s}-1} I_{\alpha}\left[\bar{f}_{\alpha}\left(t_{i}, X_{t_{i}}\right)-\bar{f}_{\alpha}\left(t_{i}, Y_{t_{i}}\right)\right]_{t_{i}, t_{i+1}}\right. \\
& \left.+\left.I_{\alpha}\left[\bar{f}_{\alpha}\left(t_{i_{s}}, X_{t_{i_{s}}}\right)-\bar{f}_{\alpha}\left(t_{i_{s}}, Y_{t_{i s}}\right)\right]_{t_{i s}, s}\right|^{2} \mid \mathcal{F}_{0}\right) \\
\leq & \operatorname{cste} \int_{0}^{t} E\left(\sup _{0 \leq s \leq u}\left|X_{t_{i u}}-Y_{t_{i u}}\right|^{2} \mathcal{F}_{0}\right) d u \\
\leq & \operatorname{cste} \int_{0}^{t} Z(u) d u
\end{aligned}
$$

In the same way using the Lemma(2)and Linear growth condition(25) we get

$$
\begin{aligned}
B_{t}^{\alpha} & :=E\left(\sup _{0 \leq s \leq t}\left|\sum_{i=o}^{i_{t}-1} I_{\alpha}\left[\bar{f}_{\alpha}\left(t_{i}, X_{t_{i}}\right)\right]_{t_{i}, t_{i+1}}+I_{\alpha}\left[\bar{f}_{\alpha}\left(t_{i_{s}}, X_{t_{i_{s}}}\right)\right]_{t_{i s}, s}\right|^{2} \mid \mathcal{F}_{0}\right) \\
& \leq \operatorname{cste} \int_{0}^{t} E\left(\sup _{0 \leq s \leq u}\left|\bar{f}_{\alpha}\left(s, X_{s}\right)\right|^{2} \mid \mathcal{F}_{0}\right) d u \\
& \leq \operatorname{cste}^{\psi(\alpha)} \int_{0}^{t} E\left(\sup _{0 \leq s \leq u}\left(1+\left|X_{s}\right|^{2} \mid \mathcal{F}_{0}\right) d u\right. \\
& \leq \operatorname{cste}^{\psi(\alpha)}\left(t+\int_{0}^{t} E\left(\sup _{0 \leq s \leq u}\left|X_{s}\right|^{2} \mid \mathcal{F}_{0}\right) d u\right)
\end{aligned}
$$

where $\psi(\alpha)=\left\{\begin{array}{ll}2 l(\alpha)-2 & : l(\alpha)=n(\alpha) \\ l(\alpha)+n(\alpha)-1 & : l(\alpha) \neq n(\alpha)\end{array}\right.$ So for $\alpha \in \mathcal{B}\left(\mathcal{A}_{\gamma}\right)$, we have $l(\alpha) \geq \gamma+1$ if $l(\alpha)=n(\alpha)$ and $l(\alpha)+n(\alpha) \geq 2 \gamma$ if $l(\alpha) \neq n(\alpha)$ so that $\psi(\alpha) \geq 2 \gamma$. Therefore applying Lemma(3) together with the above result we have

$$
B_{t}^{\alpha} \leq \operatorname{Cste}^{2 \gamma}\left(1+\left|X_{0}\right|^{2}\right)
$$


Now let us analyze the last term

$$
\begin{aligned}
& D_{t}:=E\left(\sup _{0 \leq s \leq t}\left|\int_{0}^{s}\left\{J\left(X_{t_{i_{u}}-}\right)-J\left(Y_{t_{i_{u}}-}\right)\right\} d N_{u}\right|^{2} \mid \mathcal{F}_{0}\right) \\
& =E\left(\sup _{0 \leq s \leq t} \mid \int_{0}^{s}\left\{J\left(X_{t_{i_{u}}-}\right)-J\left(Y_{t_{i_{u}}-}\right)\right\} d \tilde{N}_{u}\right. \\
& \left.+\left.\int_{0}^{s}\left\{J\left(X_{t_{i_{u}}-}\right)-J\left(Y_{t_{i_{u}}-}\right)\right\} d u\right|^{2} \mid \mathcal{F}_{0}\right) \\
& \leq 2 E\left(\sup _{0 \leq s \leq t}\left|\int_{0}^{s}\left\{J\left(X_{t_{i_{u}}}\right)-J\left(Y_{t_{i_{u}}-}\right)\right\} d \tilde{N}_{u}\right|^{2} \mid \mathcal{F}_{0}\right) \\
& +2 E\left(\sup _{0 \leq s \leq t}\left|\int_{0}^{s}\left\{J\left(X_{t_{i_{u}}-}\right)-J\left(Y_{t_{i_{u}}-}\right)\right\} d u\right|^{2} \mid \mathcal{F}_{0}\right) \\
& \leq 8 E\left(\int_{0}^{t}\left|J\left(X_{t_{i_{u}}-}\right)-J\left(Y_{t_{i_{u}}-}\right)\right|^{2} d u \mid \mathcal{F}_{0}\right) \begin{array}{l}
\text { applying Doob's inequality } \\
\text { and Itô's isometry for jump }
\end{array} \\
& +2 \lambda E\left(\int_{0}^{t}\left|J\left(X_{t_{i_{u}}-}\right)-J\left(Y_{t_{i_{u}}-}\right)\right|^{2} d u \mid \mathcal{F}_{0}\right) \\
& \leq \text { Cste } \int_{0}^{t} Z(u) d u \text {. }
\end{aligned}
$$

Then from the results (67), (69) and (70) we have for $t \in[o, T]$

$$
Z(t) \leq \operatorname{cste}\left\{E\left(\left|x_{0}-Y_{0}\right|^{2}\right)+\Delta^{2 \gamma}\left(1+\left|X_{0}\right|^{2}\right)+\int_{0}^{t} Z(u) d u\right.
$$

Therefore for $t=T$ we have

$$
Z(T) \leq \operatorname{cste}\left\{E\left(\left|x_{0}-Y_{0}\right|^{2}\right)+\Delta^{2 \gamma}\left(1+\left|X_{0}\right|^{2}\right)+\int_{0}^{T} Z(u) d u\right.
$$

Using the Assumptions(22) and applying the Gronwall inequality we obtain

$$
\begin{aligned}
Z(T) & \leq K \Delta^{2 \gamma}+\int_{0}^{T} Z(u) d u \\
& \leq K \Delta^{2 \gamma} \text { where } K \text { is a positive constant }
\end{aligned}
$$

Finally we get the desired result that concludes the proof as

$$
\sqrt{E\left(\sup _{0 \leq s \leq T}\left|X_{s}-Y_{s}\right|^{2} \mid \mathcal{F}_{0}\right)}=\sqrt{Z(T)} \leq K \Delta^{\gamma}
$$

\section{Numerical Results}

In this section we present some numerical results based on the Merton jump diffusion model as defined in Eq(6), where the jump's magnitude $Y_{j}, j=1 \ldots$ is assumed i.i.d and log-normal distributed with mean $\mu_{j}$ and standard deviation $\sigma_{j}$. We use a Monte-Carlo approach, we simulate the process, using the JAD scheme, defined in Eq(11), and compare it with the exact path. Considering the discretization time $0=t_{0}<t_{1}<\ldots<t_{N}=T$, combined with the jumps time $\tau_{i}, i=1 \ldots$, the jump adapted scheme $Y^{\Delta}=\left\{Y_{t_{i}}, i=0, \ldots, N\right\}$ of the process $X$ is :

$$
Y_{t_{i+1}}=Y_{t_{i+1}-}+\int_{t_{i}}^{t_{i+1}} J\left(Y_{t_{i+1}-}\right) d N_{t}
$$

where

$$
Y_{t_{i+1}-}=Y_{t_{i}}+\mu Y_{t_{i}} h+\sigma Y_{t_{i}} \sqrt{h} \varepsilon_{i}+\frac{1}{2} \sigma^{2} Y_{t_{i}} h\left(\varepsilon_{i}^{2}-1\right)
$$

For the following set parameters values $\mu=2$, sigma $=1 ; \lambda=1, \mu_{j}=0, \operatorname{sigma}_{j}=2, T=1$; we obtained: 
- Number of iterations $\mathrm{N}=100$ and step size $\mathrm{h}=1 / 100$

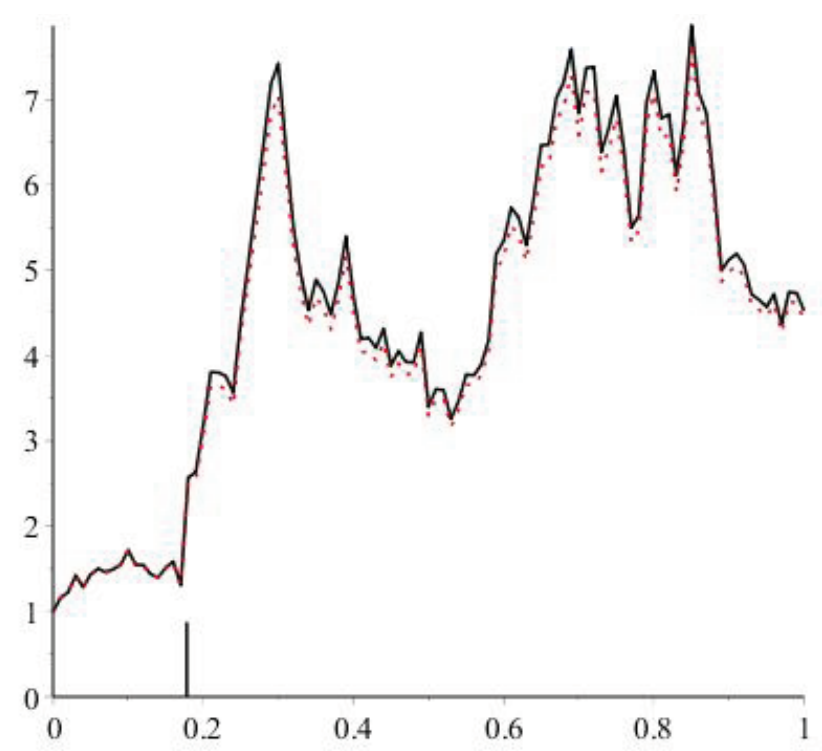

Exact path $\cdots \cdots$ JAD path

- Number of iterations $\mathrm{N}=1000$ and step size $\mathrm{h}=1 / 1000$

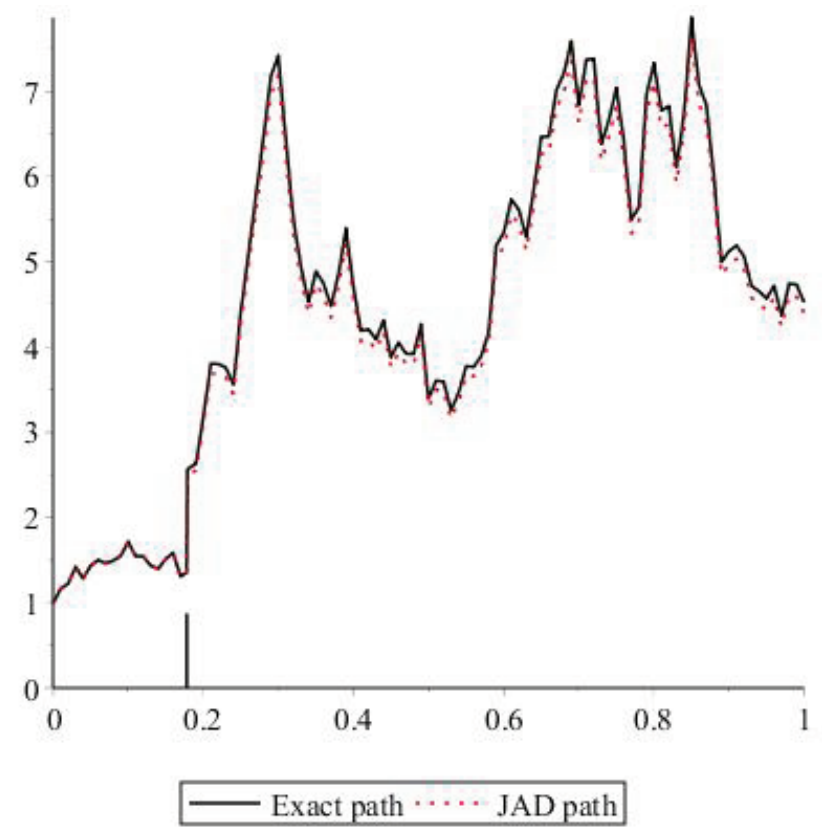

\section{Conclusion}

Through this paper we have presented the Jump adapted discretization scheme for jump diffusion with numerical on Merton Jump diffusion process. This jump discretization is more tractable since it avoids the computation of multi stochastic integral involving Poisson measure and Wiener process. The jump adapted discretization scheme has been proved to converge strongly with order one to the exact solution for a non dependent mark jump.

\section{Acknowledgements}

The completion of this research thesis has been possible through the help of many individuals who gave me their support. I thank all of them and a special thank to my wife and my son. 


\section{References}

Aitt-Sahalia, Y., Fan, J., \& Peng, H. (2009). Nonparametric transition-based tests for jump diffusions. Journal of the American Statistical Association, 104(487), 1102-1116. https://doi.org/10.1198/jasa.2009.tm08198

Aït-Sahalia, Y., \& Yu, J. (2006). Saddlepoint approximations for continuous-time markov processes. Journal of Econometrics, 134(2), 507-551. https://doi.org/10.1016/j.jeconom.2005.07.004

Akgiray, V., \& Booth, G. G. (1988). Mixed diffusion-jump process modeling of exchange rate movements. The Review of Economics and Statistics, 70(4), 631. https://doi.org/ 10.2307/1935826

Bergstrom, A. R. (1966). Survey of continuous time econometrics. [s.n.]. Black, F., \& Scholes, M. (1973). The pricing of options and corporate liabilities. Journal of Political Economy, 81(3), 637. https://doi.org/10.1086/260062

Bruti-Liberati, N., \& Platen, E. (2007). Strong approximations of stochastic differential equations with jumps. Journal of Computational and Applied Mathematics, 205(2), 982-1001. https://doi.org/10.1016/j.cam.2006.03.040

Cox, J. C., Ingersoll, J. E., \& Ross, S. A. (1985). A theory of the term structure of interest rates. Econometrica, 53(2), 385. https://doi.org/10.2307/1911242

Elerian, O. (1998). A note on the existence of a closed-form conditional transition density for the milstein scheme. In Economics discussion paper 1998- w18,.

Glasserman, P. (2004). Monte carlo methods in financial engineering. Springer.

Glasserman, P., \& Kou, S. G. (2003). The term structure of simple forward rates with jump risk. Mathematical Finance, 13(3), 383-410. https://doi.org/10.1111/1467-9965.00021

Glasserman, P., \& Merener, N. (2004). Convergence of a discretization scheme for jump-diffusion processes with statedependent intensities. Proceedings of the Royal Society A: Mathematical, Physical and Engineering Sciences, 460(2041), 111-127. doi: 10.1098/rspa.2003.1237

Gollnick, H., Houthakker, H. S., \& Taylor, L. D. (1968). Consumer demand in the united states, 1929-1970. Analyses and projections. Econometrica, 36(1), 203. https://doi.org/10.2307/1909620

Johannes, M. (2004). The statistical and economic role of jumps in continuous-time interest rate models. The Journal of Finance, 59(1), 227-260. https://doi.org/10.1111/j.1540-6321.2004.00632.x

Kloeden, P., \& Platen, E. (1992). Numerical solution of stochastic differential equations. Springer.

Lo, A.W. (1988). Maximum likelihood estimation of generalized it processes with discretely sampled data. Econ Theory, 4(02), 231-247. https://doi.org/10.1017/s0266466600012044

Merton, R. C. (1976). Option pricing when underlying stock returns are discontinuous. Journal of Financial Economics, 3(1-2), 125-144. https://doi.org/10.1016/0304-405x(76)90022-2

Merton, R. C. (1980). On estimating the expected return on the market. Journal of Financial Economics, 8(4), 323-361. https://doi.org/10.1016/0304-405x(80)90007-0

Milstein, G. N. (1979). A method of second-order accuracy integration of stochastic differential equations. Theory of Probability ; $\mathcal{E}$ Its Applications, 23(2), 396-401. https://doi.org/10.1137/1123045

Tse, Y. K., Zhang, X., \& Yu, J. (2004). Estimation of hyperbolic diffusion using the markov chain monte carlo method. Quantitative Finance, 4(2), 158-169. https://doi.org/ 10.1080/14697680400000020

Vasicek, O. (1977). An equilibrium characterization of the term structure. Journal of Financial Economics, 5(2), 177-188. https://doi.org/10.1016/0304-405x(77)90016-2

$\mathrm{Yu}, \mathrm{J}$. (2007). Closed-form likelihood approximation and estimation of jump-diffusions with an application to the realignment risk of the chinese yuan. Journal of Econometrics, 141(2), 1245-1280.

https://doi.org/10.1016/j.jeconom.2007.02.003

\section{Copyrights}

Copyright for this article is retained by the author(s), with first publication rights granted to the journal.

This is an open-access article distributed under the terms and conditions of the Creative Commons Attribution license (http://creativecommons.org/licenses/by/4.0/). 